\title{
Sistema GaitGrabber na captação de dados cinemáticos durante a marcha
}

\author{
Renata Noce Kirkwood ${ }^{1}$ \\ Renan Alves Resende ${ }^{2}$ \\ Scott Alexander Kirkwood ${ }^{3}$ \\ Felipe Cândido Gonçalves de Oliveira ${ }^{4}$ \\ Márcia Luciane Drumond das Chagas e Vallone ${ }^{4}$ \\ ${ }^{1}$ Departamento de Fisioterapia, Escola de Educação Física Fisioterapia e Terapia Ocupacional, \\ Universidade Federal de Minas Gerais, Belo Horizonte, MG, Brasil \\ ${ }^{2}$ Programa de Pós-Graduação em Ciências da Reabilitação, \\ Escola de Educação Física, Fisioterapia e Terapia Ocupacional, \\ Universidade Federal de Minas Gerais, Belo Horizonte, MG, Brasil \\ ${ }^{3}$ Engenheiro de Software, Google, Canadá \\ ${ }^{4}$ Instituto de Ciências Biológicas e da Saúde, Departamento de Fisioterapia, \\ Pontifícia Universidade Católica de Minas Gerais, Belo Horizonte, MG, Brasil
}

\begin{abstract}
Resumo: O objetivo do presente estudo foi desenvolver e testar a confiabilidade e validade do Sistema GaitGrabber na obtenção de dados cinemáticos no plano sagital durante a marcha. Dezoito indivíduos participaram do estudo de confiabilidade e 28 do estudo de validade concorrente. O Sistema Qualisys ProReflex foi usado como padrão ouro. O GaitGrabber calcula os ângulos relativos de quadril, joelho e tornozelo no plano sagital. O Coeficiente de Correlação Intraclasse (CCl) foi usado para comparação das médias dos picos angulares entre visitas. Para a validade, foi aplicada a análise de componentes principais. Os $\mathrm{CCl}$ variaram de moderados a excelentes e a validade do sistema foi comprovada para o tornozelo. Foi demonstrada diferença significativa na amplitude de movimento de quadril e joelho, a qual foi atribuída a características técnicas dos instrumentos. O Sistema GaitGrabber é válido e confiável e pode ser usado na clínica para análise cinemática da marcha no plano sagital.
\end{abstract}

Palavras-chave: Validade. Confiabilidade. Marcha. Cinemática.

\section{GaitGrabber system on kinematic gait analyses}

Abstract: The purpose of this study was to develop and test the validity and reliability of the GaitGrabber System in measuring kinematic variables in the sagittal plane during gait. Eighteen individuals participated in the reliability study and 28 in the concurrent validity study. The Qualisys Pro-Reflex System used as a gold standard reference system. The GaitGrabber calculates the relative angles at the hip, knee and ankle in the sagittal plane. The Intraclass Correlation Coefficient (ICC) was used to compare the average of the angular peaks between visits. The principal component analysis was used to test the validity of the system. The ICC ranged from moderate to excellent and the validity of the system was proved for the ankle. There were significant differences in the range of motion for the hip and knee joints which were attributed to different instrumental characteristics. The GaitGrabber system is valid and reliable and can be clinically used to analyze kinematics during gait in the sagittal plane.

Keywords: Validity. Reliability. Gait. Kinematics.

\section{Introdução}

Nos últimos anos, a tecnologia otimizou o estudo da análise do movimento. Esse avanço tecnológico atingiu principalmente o estudo da marcha, gerando dados computadorizados mais precisos e fidedignos (ASTEPHEN et al., 2008; RESENDE et al., 2010). Os dados cinemáticos descrevem o movimento e incluem variáveis como deslocamento, velocidade e aceleração angular e são obtidos por equipamentos como a cinematografia e sistemas automatizados mais avançados como o Qualisys, Optotrak e Vicon (RICHARDS, 1999; $\underline{\text { CAPPOZO }}$ et al., 2005). Entretanto, o custo desses sistemas torna praticamente inviável a sua compra, obrigando pesquisadores e clínicos a fazerem uso da análise observacional.

A literatura tem demonstrado que a qualidade das avaliações observacionais depende muito da habilidade do clínico (HILLMAN et al., 2010). Somado a isso, vários estudos vêm 
demonstrando problemas na validade $e$ fidedignidade dos dados obtidos por meio de observação visual (HILLMAN et al., 2010; MCGINLEY et al., 2003).

A análise da marcha feita por meio de sistema de vídeo de fácil manuseio e baixo custo operacional tem sido sugerida como uma fonte adicional na melhora dos métodos usuais da análise observacional (ARAÚJO et al., 2009; EASTLACK et al., 1991; WALL et al., 2000; $\underline{\text { KREBS }}$ et al., 1985). Eastlac e colaboradores (1991), usando uma câmera de vídeo, avaliaram a confiabilidade intra-examinadores das medidas têmporo-espaciais da marcha de indivíduos com artrite reumatóide. Os resultados indicaram que as avaliações dos examinadores foram parcialmente reprodutíveis e que mais padronizações para a análise de vídeo são necessárias. Na tentativa de padronizar as medidas de vídeo, Wall e colaboradores (2000) desenharam uma grade de papel sobre o chão para a determinação da posição do pé durante a fase de apoio da marcha. O paciente era solicitado a andar sobre a grade de papel sendo ao mesmo tempo filmado. Entretanto, o desenvolvimento de métodos mais padronizados ainda é necessário para favorecer a reprodutibilidade da medida (ARAÚJO et al., 2009; EASTLACK et al., 1991).

Um aprimoramento seria proporcionar que as imagens obtidas fossem lidas por um software capaz de detectar os segmentos durante a deambulação (KYRIAZIS; RIGAS, 2002). Nesse contexto, BARROS e colaboradores (1999) desenvolveram e avaliaram o sistema Digital Vídeo for Biomechanics for Windows 32 bits, Dvideow, para análise cinemática tridimensional de movimentos humanos, baseado em registros estereoscópicos de vídeo. A implantação de um sistema simples como este permite maior flexibilidade na seleção dos movimentos a serem estudados, diminui as restrições ao ambiente de coleta e permite a pesquisadores e clínicos acesso a um sistema de análise de marcha de fácil manuseio e de baixo custo operacional.

Dessa forma, o objetivo do presente estudo foi desenvolver e testar a confiabilidade e validade do sistema GaitGrabber na análise temporal, espacial e no deslocamento angular das articulações do quadril, joelho e complexo tornozelo/pé no plano sagital da marcha.

\section{Método}

A amostra foi composta por 28 indivíduos para o estudo de validade e 18 indivíduos para o estudo de confiabilidade. A amostra foi composta por homens e mulheres saudáveis voluntários. Os critérios de inclusão foram não ter sofrido lesões ou qualquer tipo de cirurgia de membros inferiores, déficits visuais significativos e anormalidades musculares neurológicas. $\mathrm{O}$ critério de exclusão foi relatar desconforto durante qualquer procedimento do estudo. Todos os participantes assinaram 0 termo de consentimento livre e esclarecido aprovado pelo Comitê de Ética em Pesquisa da Universidade Federal de Minas Gerais (ETIC 298/03).

\section{Sistema GaitGrabber}

O sistema GaitGrabber foi escrito em linguagem $\mathrm{C}_{+}+$e Python com interface simples para o usuário. No laboratório foi montada uma passarela com um piso emborrachado antiderrapante de $7 \mathrm{~m}$ de comprimento por $1 \mathrm{~m}$ de largura. Uma câmera de vídeo digital Sony DCR-TRV/950 (Sony Electronics Inc.) com frequência de captura de $30 \mathrm{~Hz}$, foi posicionada em um tripé a $38 \mathrm{~cm}$ de altura (distância do centro da lente ao chão) e a $90^{\circ}$ em relação ao solo. Essa posição da câmera, a 3,5 m do centro da passarela, permitia a visualização do participante durante um ciclo da marcha. Além disso, o tripé da câmera e o piso emborrachado de 1 metro de largura onde os voluntários foram solicitados a deambular foram sistematicamente alinhados com marcações realizadas no piso do laboratório para garantir o alinhamento da câmera com o plano sagital de movimento.

A câmera encontrava-se conectada diretamente ao computador e por meio do software VirtualDub de captura, as imagens foram obtidas. Para determinar a posição dos segmentos pelve, coxa, perna e tornozelo/pé, marcas passivas de bolas de isopor brancas partidas ao meio, de $3 \mathrm{~cm}$ de diâmetro, foram afixadas em sete proeminências ósseas. $\mathrm{Na}$ pelve, o ponto mais alto da crista ilíaca e a espinha ilíaca ântero-superior; na coxa no trocânter maior e epicôndilo lateral do fêmur e na perna e complexo tornozelo/pé, na cabeça da fíbula, maléolo lateral e na cabeça do $5^{\circ}$ metatarso. Para evitar contrastes de luz, uma cortina preta foi colocada na parede logo atrás da passarela. As mulheres vestiam short e blusa de 
malha preta e os homens short preto. Todos os participantes deambularam descalços.

Foram coletadas seis passadas e os arquivos salvos com extensão AVI. Em seguida eram abertos no programa VirtualDub, programa para captação e processamento de vídeos disponível sem custo na internet (www.virtualdub.com), no qual os arquivos eram filtrados com filtro passa baixa de $6 \mathrm{~Hz}$ para remover artefatos de movimento e salvos em vários arquivos com extensão bitmap (BMP). Primeiramente, o evento de interesse, contato do calcanhar direito ao próximo contato do mesmo calcanhar, delimitando uma passada, foi definido por meio de um footswitch, que é um transdutor de força que quando saturado emite um sinal analógico permitindo identificar contato e perda de contato do pé com solo. O footswitch foi fixado abaixo do calcâneo dos indivíduos, os quais deambularam descalços. No sistema GaitGrabber, quando o footswitch era pressionado, um led infravermelho, posicionado entre a passarela e câmera de vídeo, acendia permitindo que a imagem fosse vista e gravada pela câmera. O programa VirtualDub possui recurso que permite cortar a imagem, que nesse caso, tinha como ponto de referência a luz led que acionava ao contato e apagava quando o indivíduo perdia o contato do pé com o chão.

Em seguida, realizava-se a varredura entrelaçada para a montagem dos quadros de movimento. Na varredura, as linhas ímpares eram escaneadas primeiro e depois as linhas pares. Um conjunto de linhas (pares e ímpares) formam um campo e dois campos formavam um quadro. A cada $1 / 30$ segundo era formado um quadro da imagem.

O software do sistema GaitGrabber lê cada arquivo BMP e acha automaticamente as marcas passivas localizadas sobre as proeminências ósseas, pois possui um recurso de sensibilidade para detectar as marcas passivas de cores brancas que ressaltam no ambiente escuro. $O$ usuário pode especificar o valor dessa sensibilidade, o que facilita a captura da posição de cada marca em cada um dos quadros de movimento. Nos quadros onde o sistema não encontra a marca 0 centro da mesma pode ser localizado manualmente.

Para o cálculo do ângulo relativo das articulações do quadril (ângulo formado entre o eixo longitudinal das marcas da pelve e coxa), joelho (marcas na coxa e perna) e tornozelo/pé (marcas no tornozelo/pé ao antepé) uma filmagem de 1 segundo era obtida com 0 indivíduo em posição ortostática para determinar o ponto neutro das articulações. O sistema GaitGrabber subtrai todas as medidas angulares do ciclo da marcha da posição neutra, obtendo assim 0 ângulo relativo das articulações em relação à posição estática. Foi adotado o seguinte sistema de convenção: flexão do quadril e joelho e dorsiflexão do tornozelo foram considerados ângulos positivos e extensão do quadril e joelho e flexão plantar do tornozelo ângulos negativos.

Para o cálculo da velocidade da marcha e do tamanho da passada, um sistema de calibração montado com duas hastes de madeira fixas a uma placa, de forma que permanecessem em posições verticais, foram usadas. Duas bolas de isopor, partidas ao meio, foram afixadas na extremidade superior e inferior de cada haste. A distância vertical entre as bolas de isopor em cada haste era de $1,125 \mathrm{~m}$ e paralelamente a distância horizontal era de 2,44 m. As hastes com as marcas de isopor eram filmadas e essas distâncias eram adicionadas ao programa GaitGrabber. O programa usa essa informação para determinar quantos centímetros em pixels existem horizontalmente e verticalmente entre as bolas fixas nas hastes de madeira. Dessa forma, a distância horizontal em centímetros entre a marca do maléolo lateral direito no momento em que o pé tocava o solo até a mesma marca no momento em que a mesmo pé tocava o solo novamente poderia ser computada, gerando a medida do comprimento da passada. A velocidade da marcha foi determinada por meio da divisão do comprimento do passo pelo número de quadros dividido por 30 segundos. As variáveis velocidade da marcha e comprimento do passo não foram controladas durante a realização da coleta de dados, mas calculadas durante o processamento dos dados. O sistema GaitGrabber será disponibilizado na internet, não tem fins lucrativos e possuirá código aberto para aprimoramentos do programa. Também será disponibilizado um manual com as instruções do programa e alguns dados para exemplificar.

\section{Sistema Qualisys Pró-Reflex}

Como sistema padrão ouro foi utilizado o sistema de cinemetria Qualisys Pro-Reflex MCU 
240 (QUALISYS MEDICAL AB, $411 \quad 12$ Gothenburg, Suécia) composto por seis câmeras. A calibração do sistema foi realizada de acordo com o manual do fabricante e a frequência de captação dos dados foi de $120 \mathrm{~Hz}$.

Para a construção do modelo biomecânico e processamento dos dados cinemáticos foi usado o software Visual 3D e aplicada a sequência de Cardan para obtenção dos dados angulares do quadril, joelho e complexo tornozelo/pé durante a marcha no plano sagital. O método empregado pelo Sistema Qualisys e Visual 3D já foi publicado em detalhes na literatura (ARAÚJO et al., 2009).

Para a definição dos eventos, contato inicial ao próximo contato inicial do mesmo pé, delimitando uma passada, foi usado o mesmo footswitch que se encontrava conectado por meio de cabo coaxial ao sistema de captura analógica que integra o Qualisys, permitindo assim a coleta simultânea dos dados.

\section{Procedimentos}

\section{Confiabilidade teste-reteste do} Sistema GaitGrabber

As coletas ocorreram no Laboratório de Análise de Movimento do Departamento de Fisioterapia da Universidade Federal de Minas Gerais. Para avaliar a confiabilidade do sistema GaitGrabber, 18 indivíduos saudáveis foram convidados a participar do estudo de acordo com os critérios mencionados. Inicialmente foram coletados dados antropométricos seguido da coleta dos dados da marcha. Um examinador foi devidamente treinado para a realização das coletas e o mesmo examinador foi responsável por realizar todas as coletas do estudo de confiabilidade do sistema. Durante a coleta dos dados da marcha, os indivíduos foram solicitados a andar em sua velocidade habitual para frente em direção à câmera número seis do Sistema Qualisys, a qual estava posicionada no final da passarela. $O$ membro dominante foi utilizado para a coleta dos dados sendo obtidas em média seis passadas por indivíduo. As coletas foram realizadas em duas visitas, com intervalo de uma semana. Esse procedimento foi necessário para permitir comparação entre os dados e calcular os coeficientes de confiabilidade teste-reteste.

\section{Validade do Sistema GaitGrabber}

Para a validade concorrente do sistema, participaram 28 indivíduos saudáveis de acordo com os critérios mencionados. As características antropométricas foram obtidas seguidas da coleta dos parâmetros da marcha. O mesmo examinador foi devidamente treinado e responsável por realizar todas as coletas simultâneas com os dois sistemas de análise de movimento. As curvas temporais foram coletadas simultaneamente pelo sistema GaitGrabber e Qualisys Pró-Reflex, possibilitado por meio de um trigger, que ao ser acionado iniciava a coleta dos dois sistemas. É importante ressaltar que abordagens bidimensionais (2D) possuem limitações técnicas e computacionais quando comparadas com abordagens tridimensionais (3D) (ALLARD et al., 1995; CAPPOZZO. 1984), o que restringe a sua utilização apenas para a mensuração de movimentos de grande amplitude como os encontrados no plano sagital. O membro dominante foi $o$ escolhido e foram obtidas seis passadas por indivíduo.

\section{Análise dos dados}

As características dos indivíduos foram descritas de acordo com a média e o desvio padrão. Para a confiabilidade foi usado 0 Coeficiente de Correlação Intraclasse (CCl) (PORTNEY; WATKINS, 2000) para os picos angulares máximos/mínimos e para a amplitude de movimento (ADM). A média da velocidade da marcha e do comprimento da passada obtidos entre visitas foram comparadas usando testes de inferência.

A validade concorrente do deslocamento angular no plano sagital foi testada usando a Análise de Componentes Principais (ACP) que sumariza as informações das variáveis do ciclo da marcha em um número pequeno de componentes que explicam a variância dos dados (JOLLIFFE, 2004). A análise resulta em coeficientes que representam características específicas da curva e os escores que são a distância que a curva de cada indivíduo está da média de uma determinada componente. Componentes com autovalores maiores que um foram escolhidos para análise (JOLLIFFE, 2004). A interpretação das componentes foi realizada por meio dos coeficientes e da comparação entre a média das curvas dos indivíduos com escores altos e baixos (DELUZIO et al., 1997; 
KIRKWOOD et al., 2011). Em seguida, os escores de cada indivíduo obtidos em cada um dos sistemas foram comparados usando teste $t$ pareado para dados com distribuição normal e Wilcoxon para dados que não apresentaram distribuição normal. Foi usado o pacote estatístico SPSS-15 e Minitab-16 com nível de significância de 0,05.

\section{Resultados}

As características dos participantes dos dois estudos estão representadas na Tabela 1. Os dados da confiabilidade estão representados na Tabela 2 com a média e o desvio padrão dos ângulos de cada articulação e suas respectivas ADM's durante as visitas e o local de ocorrência no ciclo da marcha (\%). Observa-se que os valores de $\mathrm{CCl}$ apresentaram confiabilidade excelente para os picos angulares e para as
ADM's em todas as articulações. Somente o pico de extensão do joelho apresentou confiabilidade moderada.

Tabela 1. Características dos participantes no início do estudo para os testes de validade e confiabilidade.

\begin{tabular}{ccc}
\hline Características & $\begin{array}{c}\text { Confiabilidade } \\
(\mathrm{N}=18) \\
\text { Média }(\mathrm{DP})\end{array}$ & $\begin{array}{c}\text { Validade } \\
(\mathrm{N}=28) \\
\text { Média (DP) }\end{array}$ \\
\hline Idade (anos) & $26,6(9,5)$ & $22,5(2,7)$ \\
Altura $(\mathrm{m})$ & $1,7(0,1)$ & $1,8(0,1)$ \\
Massa $(\mathrm{kg})$ & $60,0(10,0)$ & $68,3(9,9)$ \\
IMC $\left(\mathrm{kg} / \mathrm{m}^{2}\right)$ & & $22,2(2,7)$ \\
$\begin{array}{c}\text { Sexo, } \mathrm{n} \text { de } \\
\text { mulheres/total }\end{array}$ & $9 / 18$ & $7 / 28$ \\
\hline
\end{tabular}

Tabela 2. Média, desvio padrão (DP) e \% do ciclo dos dados espaço-temporais e angulares no plano sagital das articulações do quadril, joelho e complexo tornozelo/pé nas visitas 1 e 2 e o coeficiente de correlação intraclasse $(\mathrm{CCl})$ entre as visitas dos 18 participantes obtidas por meio do sistema GaitGrabber.

\begin{tabular}{|c|c|c|c|}
\hline \multirow[b]{2}{*}{ Parâmetros } & \multicolumn{2}{|c|}{ Visitas } & \multirow{2}{*}{$\begin{array}{c}\text { Confiabilidade } \\
\mathrm{CCl}\end{array}$} \\
\hline & $\begin{array}{c}\text { Visita } 1 \\
(\mathrm{~N}=18) \\
\text { Média (DP) }\end{array}$ & $\begin{array}{c}\text { Visita } 2 \\
(\mathrm{~N}=18) \\
\text { Média (DP) }\end{array}$ & \\
\hline Velocidade da marcha (m/s) & $1,3(0,1)$ & $1,3(0,1)$ & 0.96 \\
\hline Comprimento do passo (m) & $1,4(0,2)$ & $1,4(0,2)$ & 0.97 \\
\hline \multicolumn{4}{|l|}{ Quadril } \\
\hline $\begin{array}{l}\text { Máxima Flexão } \\
\text { \% ciclo }\end{array}$ & $\begin{array}{c}26,8^{\circ}\left(3,4^{\circ}\right) \\
87\end{array}$ & $\begin{array}{c}25,6^{\circ}\left(3,7^{\circ}\right) \\
87\end{array}$ & 0.91 \\
\hline $\begin{array}{l}\text { Máxima Extensão } \\
\% \text { ciclo }\end{array}$ & $\begin{array}{c}-12,6^{\circ}\left(2,7^{\circ}\right) \\
55\end{array}$ & $\begin{array}{c}-13,1^{\circ}\left(2,7^{\circ}\right) \\
54\end{array}$ & 0.79 \\
\hline $\begin{array}{l}\text { ADM } \\
\text { Joelho }\end{array}$ & $39,5\left(4,4^{\circ}\right)$ & $38,6\left(3,4^{\circ}\right)$ & 0.94 \\
\hline $\begin{array}{l}\text { Máxima Flexão } \\
\text { \% ciclo }\end{array}$ & $\begin{array}{c}58,6^{\circ}\left(4,0^{\circ}\right) \\
74\end{array}$ & $\begin{array}{c}59,0^{\circ}\left(5,0^{\circ}\right) \\
73\end{array}$ & 0.77 \\
\hline $\begin{array}{l}\text { Máxima Extensão } \\
\text { \% ciclo }\end{array}$ & $\begin{array}{c}-2,7^{\circ}\left(2,6^{\circ}\right) \\
99\end{array}$ & $\begin{array}{c}-2,7^{\circ}\left(3,0^{\circ}\right) \\
99\end{array}$ & 0.65 \\
\hline ADM & $61,3^{\circ}\left(3,9^{\circ}\right)$ & $61,7^{\circ}\left(3,6^{\circ}\right)$ & 0.81 \\
\hline \multicolumn{4}{|l|}{ Tornozelo } \\
\hline $\begin{array}{l}\text { Máxima Dorsiflexão } \\
\text { \% ciclo }\end{array}$ & $\begin{array}{c}9,3^{\circ}\left(2,5^{\circ}\right) \\
44\end{array}$ & $\begin{array}{c}10,1^{\circ}\left(3,2^{\circ}\right) \\
46\end{array}$ & 0.84 \\
\hline $\begin{array}{l}\text { Máxima Flexão Plantar } \\
\text { \% ciclo }\end{array}$ & $\begin{array}{c}-15,4^{\circ}\left(4,4^{\circ}\right) \\
66\end{array}$ & $\begin{array}{c}-14,6^{\circ}\left(4,4^{\circ}\right) \\
66\end{array}$ & 0.87 \\
\hline ADM & $24,6^{\circ}\left(5,7^{\circ}\right)$ & $24,7^{\circ}\left(4,9^{\circ}\right)$ & 0.92 \\
\hline
\end{tabular}

$\mathrm{ADM}=$ amplitude de movimento

A Figura $1 \mathrm{~A}$ mostra que $\mathrm{O}$ deslocamento angular obtido entre visitas foi similar em forma $e$ magnitude. A Figura 1B mostra a média ( \pm DP) dos 18 participantes nas duas visitas.
Em relação aos resultados da validade entre sistemas, a Tabela 3 mostra a média $( \pm \mathrm{DP})$ da velocidade da marcha e do comprimento da passada. Observa-se que não houve diferença significativa entre medidas $(p>0,05)$. 
A) Visita 1

Visita $2---$
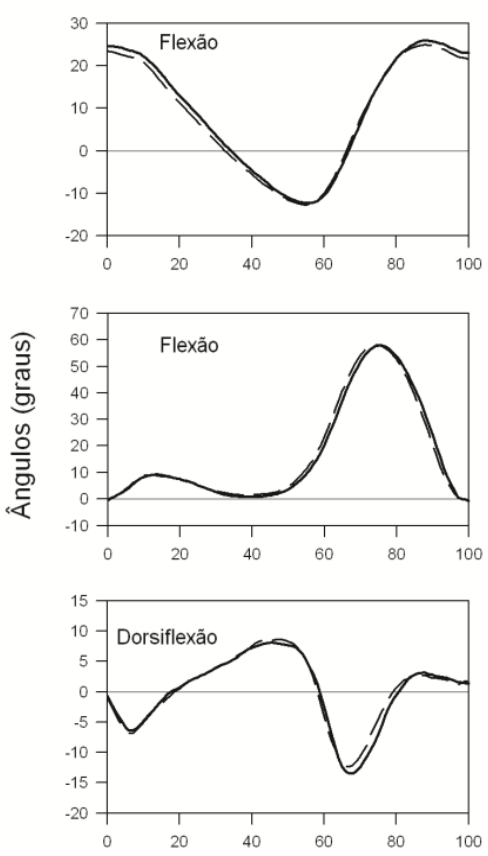

B) Média visita 1 e 2 + +- DP - - -
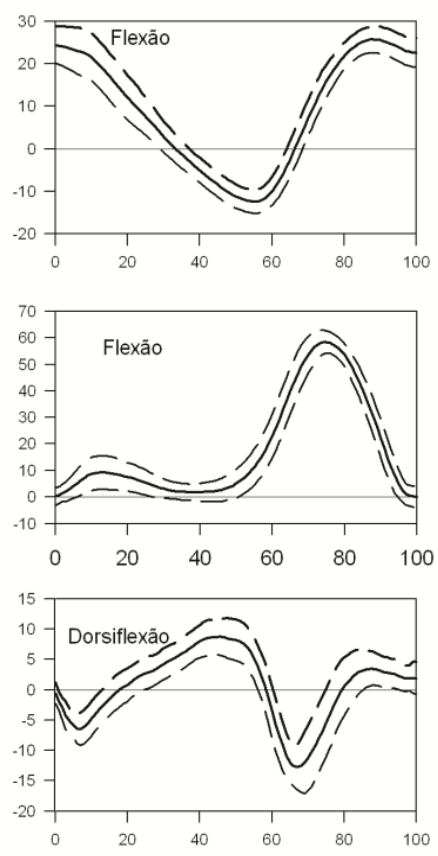

$\%$ Ciclo da Marcha

Figura 1. A) Padrão angular do quadril, joelho e complexo tornozelo/pé no plano sagital entre as visitas 1 e 2 obtidas pelo sistema GaitGrabber $(\mathrm{N}=18)$. B) Média das duas visitas, mais ou menos um desvio padrão (+/-DP) das angulações do quadril, joelho e tornozelo/pé obtidas pelo sistema GaitGrabber.

Tabela 3. Média, desvio padrão e p-valor dos parâmetros espaço-temporais obtidos pelos sistemas GaitGrabber e Qualisys Pró-Reflex (N=28).

\begin{tabular}{cccc}
\hline \multirow{2}{*}{ Parâmetros } & \multicolumn{2}{c}{ Sistemas } & \\
\cline { 2 - 3 } & $\begin{array}{c}\text { GaitGrabber } \\
\mathrm{N}=28 \\
\text { Média }(\mathrm{DP})\end{array}$ & $\begin{array}{c}\text { Qualisys } \\
\mathrm{N}=28 \\
\text { Média (DP) }\end{array}$ & P-valor \\
\hline Velocidade da marcha $(\mathrm{m} / \mathrm{s})$ & $1,2(0,2)$ & $1,1(0,1)$ & $0,290^{\underline{\mathrm{a}}}$ \\
Comprimento do passo $(\mathrm{m})$ & $1,4(0,2)$ & $1,4(0,2)$ & $0,064^{\mathrm{b}}$ \\
\hline
\end{tabular}

${ }^{a}$ Usado teste Wilcoxon; ${ }^{b}$ usado teste t pareado; signicativo $p<0,05$.

A Figura 2 mostra a média do deslocamento angular no plano sagital das articulações do quadril (A), joelho (D) e complexo tornozelo/pé entre sistemas $(G)$. Observa-se que as curvas seguem um mesmo padrão, com uma tendência do sistema GaitGrabber em gerar valores maiores. A ACP das articulações do quadril, joelho e complexo tornozelo/pé resultaram em apenas uma componente com autovalores maiores que um. A CP1 do quadril explicou $84,8 \%$, a CP1 do joelho $59,2 \%$ e a CP1 do complexo tornozelo/pé $60,1 \%$ da variância dos dados. As interpretações das componentes das três articulações geraram valores positivos retratando a $A D M$ articular em todo ciclo da marcha (Figura 2B, E, H). Os gráficos da Figura 2G, $\mathrm{H}$ e I, mostram o deslocamento articular de um indivíduo com escore mais baixo, ou seja, mais distante da componente e representado por uma coleta feita com o sistema Qualisys e de um indivíduo cujo escore era o mais alto e representado por dados coletados pelo sistema GaitGrabber. Como as curvas estão distantes em termos de amplitude, confirmam a interpretação, ou seja, se houve diferença entre as medidas, essa diferença ocorreu na amplitude do movimento. 

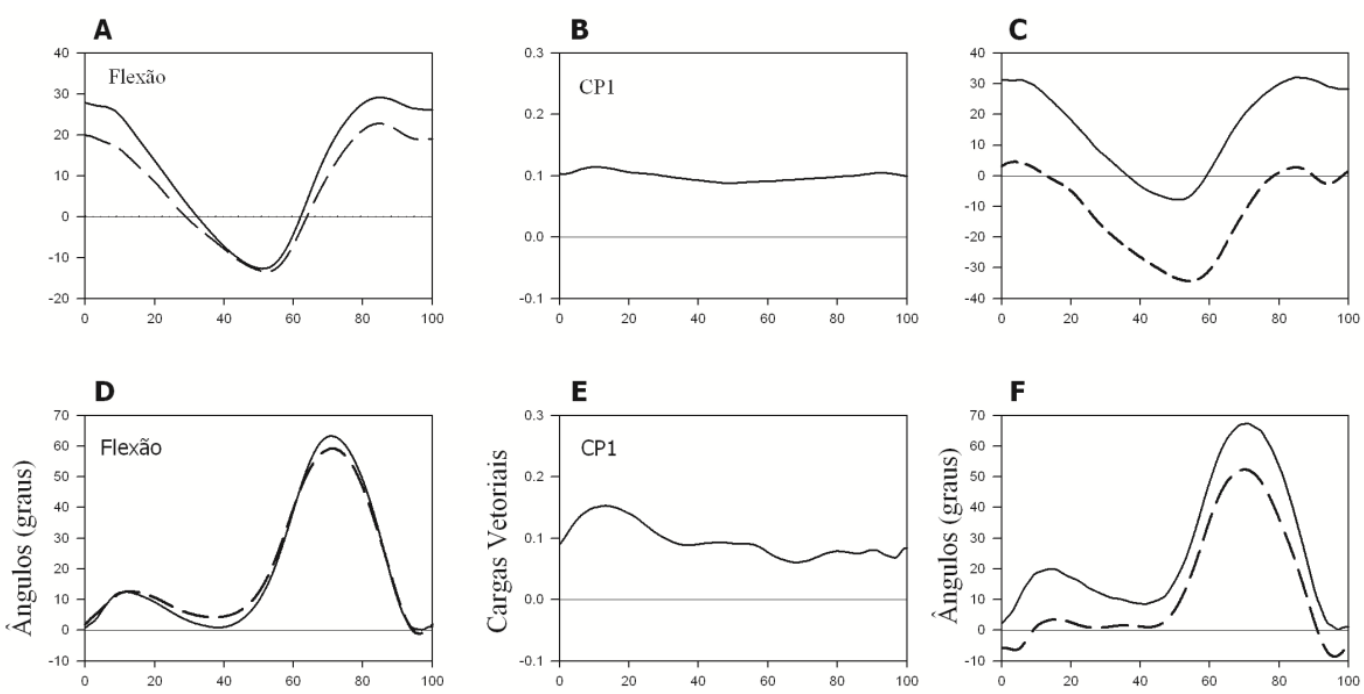

G
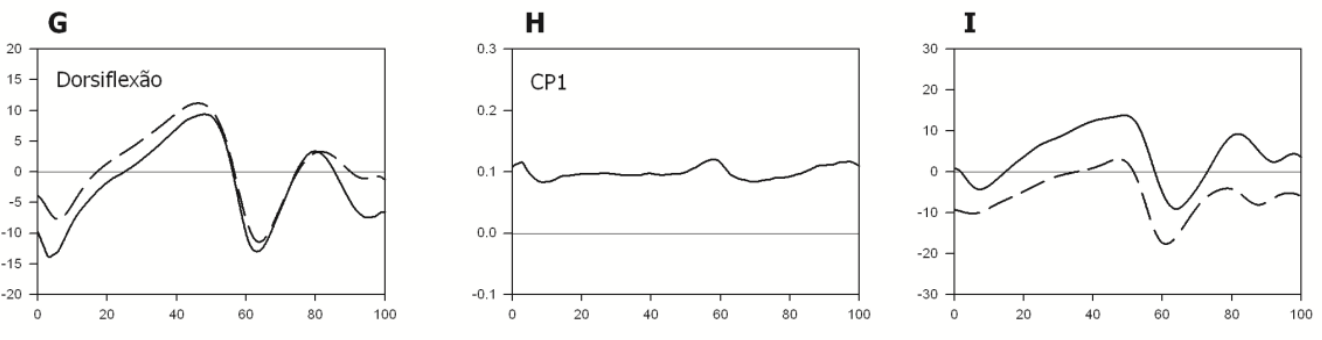

$\%$ Ciclo da Marcha

Escore alto

Escore baixo - - -

Figura 2. Média do deslocamento angular do quadril (A), joelho (D) e complexo tornozelo/pé (G) no plano sagital entre o sistema GaitGrabber (linha contínua) e o sistema Qualisys Pró-Reflex (linha pontilhada) (N= 28). Cargas vetoriais das componentes 1 da articulação do quadril (B), joelho (E) e complexo tornozelo/pé $(\mathrm{H})$. Escore mais alto (linha contínua) representado por dados angulares coletados com o sistema GaitGrabber e mais baixo (linha pontilhada), representado por dados coletados com o sistema Qualisys no quadril $(C)$, joelho $(F)$ e complexo tornozelo/pé (I).

Para determinar se as diferenças em amplitudes eram significativas, os escores de cada indivíduo obtido por cada sistema foram comparados por meio de testes de inferência. Os resultados mostraram uma diferença significativa nos escores da CP1 das articulações do quadril $(p<0,000)$ e joelho $(p<0,000)$, entretanto nenhuma diferença foi encontrada no complexo tornozelo/pé $(p=0,144)$. Portanto, os sistemas se diferenciam em termos de amplitude no ciclo das articulações do quadril e joelho. Em média, a diferença de amplitude entre sistemas no deslocamento angular do quadril foi de $5,6^{\circ}$, na articulação do joelho de $3,1^{\circ}$ e no complexo tornozelo/pé de $0,6^{\circ}$.

\section{Discussão}

O objetivo do presente estudo foi desenvolver um instrumento de captação de dados cinemáticos de baixo custo, gerando informações válidas, reproduzíveis e de fácil acesso à clínica. Nossos resultados indicam que o sistema GaitGrabber é confiável na obtenção de dados têmporo-espaciais e angulares da marcha no plano sagital. Os valores de $\mathrm{CCl}$ em sua maioria foram excelentes e a localização próxima na porcentagem do ciclo da marcha dos picos de flexão/extensão e dorsiflexão/flexão plantar encontrados entre visitas demonstram a boa reprodutibilidade do sistema.

Em relação à validade concorrente do sistema GaitGrabber, a ACP mostrou diferenças na amplitude de movimento durante todo o ciclo da marcha nas três articulações, entretanto, apenas os escores do quadril e joelho foram diferentes entre os sistemas de medida. As diferenças encontradas na amplitude do ciclo no quadril e joelho foram de $5,6^{\circ}$ e $3,1^{\circ}$, respectivamente. 
Dentre os fatores que podem justificar as diferenças entre sistemas está a localização do ponto anatômico por meio da palpação, principalmente superfícies ósseas como o ponto mais alto da crista ilíaca e o trocânter maior do fêmur (CROCE et al., 2005). Se a medida angular é baseada em marcas externas sobre proeminências ósseas, a posição das marcas é que irá definir a orientação do eixo longitudinal externo dos segmentos. Biden e colaboradores (1990) mostraram que um ligeiro deslocamento da marca passiva posteriormente ao trocânter maior ou anteriormente ao côndilo femoral lateral pode gerar diferenças na leitura angular de flexão do joelho em até $10^{\circ}$. Portanto, sistemas como o GaitGrabber que se baseiam no correto alinhamento e posicionamento da marca passiva, sofrem esse tipo erro. Outra fonte de erro está em acessar o movimento estritamente de um único plano. Por exemplo, um indivíduo visto no plano sagital pode aparentemente apresentar um determinado valor de flexão do joelho, entretanto, esse mesmo indivíduo pode estar com o membro rodado internamente. $O$ efeito da rotação interna diminui a leitura angular de flexão, pois aproxima o eixo longitudinal da coxa em relação à tíbia. Portanto, ao avaliar os movimentos estritamente em um plano, deve-se considerar a influência das possíveis rotações do membro inferior sobre os dados obtidos.

Os erros de posicionamento das marcas passivas também afetam diretamente as medidas de sistemas mais avançados como o Qualisys Pró-reflex. Nesses instrumentos, a posição das marcas externas é corrigida horizontalmente, verticalmente e lateralmente de modo a refletirem o verdadeiro centro de rotação da articulação. Os eixos longitudinais e as coordenadas são construídos a partir dessa nova marca interna. Portanto, caso a marca externa esteja mal posicionada, esse erro será transferido para a correção interna. Kirkwood e colaboradores (2004) demonstraram que técnicas não invasivas de determinação do centro de rotação do quadril são afetadas tanto pelo mau posicionamento da marca externa quanto pelo plano de movimento onde a correção é feita.

Somado a isso, sistemas de análise de movimento baseados em vídeo sofrem de um erro inerente relacionada à distorção nas lentes das câmeras. As lentes padrões captam a imagem sem distorção quando o movimento está central à lente. Entretanto, à medida que o movimento se distancia da lente, ocorrem distorções na imagem que afetam a captação das marcas nas extremidades. Podemos observar nos gráficos da Figura $2 \mathrm{~A}$ que $\mathrm{O}$ deslocamento angular do quadril entre sistemas é bem diferente nas extremidades do movimento e na Figura $2 \mathrm{~F}$ os escores se distanciam mais no início e final do deslocamento angular do joelho.

Para corrigir esse erro é necessária a calibração do sistema que consiste na captação pelas câmeras de várias marcas passivas dentro de um volume de interesse. Esse procedimento, denominado Transformação Linear Direta, tem como objetivo determinar as características geométricas e ópticas internas das câmeras e a posição e orientação das câmeras em relação às marcas externas dentro do volume calibrado ( $\underline{\mathrm{CHIARI}}$ et al., 2005). Portanto, o sistema Qualisys corrige as distorções, pois possui um sistema de calibração similar. Já o sistema GaitGrabber passa por um processo de calibração que inclui apenas o reconhecimento do tamanho das marcas e distâncias em pixels de uma marca a outra, permitindo assim o cálculo do comprimento do passo e da velocidade não havendo correção para o efeito das lentes.

Atribuímos a diferença nas amplitudes de movimento observadas entre os dois sistemas às diferenças técnicas e de qualidade, uma vez que os deslocamentos angulares da articulação do quadril e do joelho dependem dessas marcas anatômicas. No tornozelo esse erro não foi encontrado, possivelmente pelo fato das proeminências ósseas do complexo tornozelo/pé serem mais facilmente palpáveis, o que ameniza o erro de medida. No plano sagital ocorrem as maiores amplitudes de movimento, dessa forma, diferenças pequenas como as encontradas no presente estudo não podem ser consideradas erros aleatórios do sistema GaitGrabber.

Biden e colaboradores (1990) sugerem que uma das maneiras de eliminar erros constantes de posicionamento e distorção é subtrair a média total do grupo da média de cada indivíduo, o que resulta em um deslocamento angular relativo à média. Dessa maneira, removemos o erro sem alterar o padrão da curva, permitindo comparação entre curvas usando métodos estatísticos avançados como a ACP (DELUZIO; ASTEPHEN, 2007), que retratam 
verdadeiramente o padrão angular do indivíduo em todo o ciclo da marcha.

Embora as inúmeras vantagens que um sistema de fotogrametria pode oferecer, como melhor frequência de captação (120 Hz ou mais) e dados nos três planos de movimento, a viabilidade, manutenção e análise dos dados são de alto custo, tornando esse tipo de instrumento accessível apenas a grandes centros de pesquisa e de saúde. O sistema GaitGrabber tem como vantagem exatamente o baixo custo, acesso e facilidade na obtenção dos dados.

Entre as limitações do sistema GaitGrabber está a medida do deslocamento angular em apenas um plano de movimento. Sabemos da importância das informações angulares referentes aos planos de movimento frontal e transverso, portanto seria interessante que 0 sistema fosse implementado para permitir medidas em outros planos de movimento. Outro fator importante seria determinar a confiabilidade inter-examinadores do sistema e se ele é capaz de ser confiável em condições atípicas da marcha.

Em conclusão, o sistema proposto permite a obtenção do deslocamento angular da articulação do quadril, joelho e tornozelo no plano sagital de forma reproduzível e válida. Em relação aos dados espaço-temporais, as medidas obtidas pelo sistema GaitGrabber são repetívies e próximas de valores descritos na literatura (CHUNG, 2000; KERRIGAN et al., 1998). Como no plano sagital ocorrem as maiores amplitudes de movimento, a avaliação apenas nesse plano já contribuiria muito para o conhecimento das limitações de movimento durante a marcha e colaborariam para a implementação do tratamento fisioterápico. Somado a isso, o sistema apresentado é simples de ser usado e de baixo custo operacional, podendo ser utilizado como ferramenta clínica sem ônus elevado para os fisioterapeutas e demais profissionais interessados na análise de movimento. A necessidade de um sistema acessível para o estudo da marcha é essencial para a compreensão e o desenvolvimento de medidas terapêuticas mais efetivas.

\section{Referências}

ALLARD, P., STOKES, A. F., BLANCHI, J. P. Three-dimensional analysis of human movement. In: Human Kinetics, Champaign. 371p, 1995.

ARAÚJO, P. A., KIRKWOOD, R. N., FIGUEIREDO, E. M. Validity and intra- and interrater reliability of the Observational Gait Scale for children with spastic cerebral palsy. Revista

Brasileira de Fisioterapia, São Carlos, v. 13, n. 3, p. 267-273, 2009.

ASTEPHEN, J. L., DELUZIO, K. J., CALDWELL, G. E., DUNBAR, M. J. Biomechanical changes at the hip, knee, and ankle joints during gait are associated with knee osteoarthritis severity. Journal of orthopaedic research., New York, v. 26, n. 3, p. 332-341, 2008.

BARROS, R. M. L., BRENZIKOFER, R., LEITE, N. J., FIGUEROA, P. J. Desenvolvimento e avaliação de um sistema para análise cinemática tridimensional de movimentos humanos. Revista Brasileira de Engenharia Biomédica, Rio de Janeiro, v. 15, n. 1-2, p. 79-86, 1999.

BIDEN, E. et al. Gait Analysis. In: DANIEL, D. Knee Ligaments: Structure, Function, Injury, and Repair. Toronto: cap. Raven Press Ldt., p. 291-311, 1990.

CAPPOZZO, A. Gait analysis methodology. Human Movement Science, Amsterdam, v. 3, p. 27-50, 1984.

CAPPOZZO, A., CROCE, U. D., LEARDINI, A., CHIARI, L. Human movement analysis using stereophotogrammetry; Part 1: theoretical background. Gait \& Posture, Oxford, v. 21, n. 2, p. 186-196, 2005.

CHIARI, L., CROCE, U. D., LEARDINI, A., CAPPOZO, A. Human movement analysis using stereophotogrammetry; Part 2: Instrumental errors. Gait \& Posture, Oxford, 21(2):197-211, 2005.

CHUNG, T. M. Avaliação cinética e cinemática da marcha de adultos do sexo masculino. Acta Fisiátrica., São Paulo, v. 7, n. 2, p. 61-67, 2000.

CROCE, U. D., LEARDINI A., CHIARI, L., CAPPOZZO, A. Human movement analysis using stereophotogrammetry Part 4: assessment of anatomical landmark misplacement and its effects on joint kinematics. Gait \& Posture, Oxford, v. 21, n. 2, p. 226-237, 2005

DELUZIO, K. J., WYSS, U. P., ZEE, B., COSTIGAN, P., SORBIE, C. Principal component models of knee kinematics and kinetics: Normal 
vs. pathological gait patterns. Human Movement Science, Amsterdam, v. 16, p. 201-217, 1997.

DELUZIO, K. J., ASTEPHEN, J. L.

Biomechanical features of gait waveform data associated with knee osteoarthritis: an application of principal component analysis. Gait \& Posture, Oxford, v. 25, n. 1, p. 86-93, 2007.

EASTLACK, M. E., ARVIDSON, J., SNYDERMACKLER, L, DANOFF, J. V., MCGARVEY, D. L. Interrater reliability of videotaped observational gait-analysis assessments. Physical Therapy, New York, v. 71, n. 6, p. 465-472, 1991.

HILLMAN, S. J., DONALD, S. C., HERMAN, J., MCCURRACH, E., MCGARRY, A., RICHARDSON, A. M., ROBB, J. E. Repeatability of a new observational gait score for unilateral lower limb amputees. Gait \& Posture, Oxford, v. 32, n. 1, p. 39-45, 2010.

JOLLIFFE, I. T. Principal Component Analysis. 2. ed. New York: Springer, 2004.

KERRIGAN, D. C., TODD, M. K., DELLA, C. U. Gender differences in joint biomechanics during walking: normative study in young adults.

American journal of physical medicine and rehabilitation, Baltimore, v. 77 , n. 1, p. 2-7, 1998.

KIRKWOOD, R. N., CULHAM, E., COSTIGAN, P. Radiographic and non-invasive determination of the hip joint center location: Effect on hip joint angles. Revista Brasileira de Fisioterapia, São Carlos, v. 8, n. 1, p. 29-37, 2004.

KIRKWOOD, R. N., RESENDE, R. A., MAGALHÃES, C. M., GOMES, H. A., MINGOTI, S. A., SAMPAIO, R. F. Application of principal component analysis on gait kinematics in elderly women with knee osteoarthritis. Revista

Brasileira de Fisioterapia, São Carlos, v. 15, n. 1, p. 52-58, 2011.

KREBS, D. E., EDELSTEIN, J. E., FISHMAN, S. Reliability of observational kinematic gait analysis. Physical Therapy, New York, v. 65, n. 7, p. 1027-1033, 1985.

KYRIAZIS, V., RIGAS, C. Software for temporal gait data analysis. Computer methods and programs in biomedicine, Amsterdam, v. 67, p. 225-229, 2002.

MCGINLEY, J. L., GOLDIE, P. A., GREENWOOD, K. M., OLNEY, S. J. Accuracy and reliability of observational gait analysis data: Judgments of push-off in gait after stroke.

Physical Therapy, New York, v. 83, n. 2, p. 146158, 2003.
PORTNEY, L. G., WATKINS, M. P. Statistical measures of Reliability. In: MEHALIK, C.

Foundations of Clinical Research.

Applications to Practice. 2. ed. New Jersey:

Prentice-Hall, cap. 26, p. 557-586, 2000.

RESENDE, R. A., KIRKWOOD, R. N., FIGUEIREDO, E. M. Cinemática da marcha de adultos jovens: dados normativos iniciais.

Revista Terapia Manual, Londrina, v. 8, n. 39, p. 370-376, 2010.

RICHARDS, J. G. The measurement of human motion: A comparison of commercially available systems. Human Movement Science,

Amsterdam, v. 8, p. 589-602, 1999.

WALL, J. C., DEVLIN, J., KHIRCHOF, R., LACKEY, B. Measurement of step widths and step lengths: a comparison of measurements made directly from a grid with those made from a video recording. Journal of Orthopaedic and Sports Physical Therapy, Washington, v. 30, n. 7, p. 410-417, 2000.

Agradecimentos: Ao Departamento de Fisioterapia da UFMG e ao Fundo de Investimento em Pesquisa da PUC-MG pelo incentivo financeiro, e a todos os alunos que de algum modo contribuíram para a realização desse projeto.

Endereço:

Renata Noce Kirkwood

UFMG - Escola de Educação Física, Fisioterapia e Terapia/Ocupacional

Departamento de Fisioterapia

Av. Antônio Carlos 6627

Belo Horizonte MG Brasil

31270-901

Telefone: (31) 3409-4783 ou 31 9985-0707

Fax: 31 3409-4781

e-mail : renata.kirkwood@gmail.com

Recebido em: 28 de junho de 2011.

Aceito em: 3 de abril de 2012.

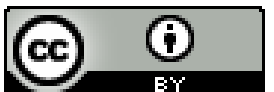

Motriz. Revista de Educação Física. UNESP, Rio Claro, SP, Brasil - elSSN: 1980-6574 - está licenciada sob Creative Commons - Atribuição 3.0 